\title{
DROP EJECTION FROM AN OSCILLATING ROD
}

\author{
E.D. Wilkes, O.A. Basaran, School of Chemical Engineering, Purdue University, West Lafayette IN 47906, USA, \\ obasaran@ecn.purdue.edu
}

\begin{abstract}
The dynamics of a drop of a Newtonian liquid that is pendant from or sessile on a solid rod that is forced to undergo time-periodic oscillations along its axis is studied theoretically. The free boundary problem governing the time evolution of the shape of the drop and the flow field inside it is solved by a method of lines using a finite element algorithm incorporating an adaptive mesh. When the forcing amplitude is small, the drop approaches a limit cycle at large times and undergoes steady oscillations thereafter. However, drop breakup is the consequence if the forcing amplitude exceeds a critical value. Over a wide range of amplitudes above this critical value, drop ejection from the rod occurs during the second oscillation period from the commencement of rod motion. Remarkably, the shape of the interface at breakup and the volume of the primary drop formed are insensitive to changes in forcing amplitude. The interface shape at times close to and at breakup is a multi-valued function of distance measured along the rod axis and hence cannot be described by recently popularized one-dimensional approximations. The computations show that drop ejection occurs without the formation of a long neck. Therefore, this method of drop formation holds promise of preventing formation of undesirable satellite droplets.
\end{abstract}

\section{INTRODUCTION}

Formation of small drops of one phase into another phase by flowing the former phase through a suitable nozzle is of great scientific interest because of widespread use of the phenomenon in practical applications. Some well known applications include mass transfer operations in solvent extraction, ${ }^{1,2}$ ink-jet printing, ${ }^{3,4}$ and measurement of dynamic surface tension, 5,6 among others.

When a liquid is fed continuously through a capillary tube at a low flow rate, a portion of the drop liquid hanging from the tip of the capillary breaks off it when the force of gravity acting on the drop becomes large enough to overcome the force of surface tension. ${ }^{7,8}$ Unfortunately, the gravitationally induced mechanism of drop breakup is not available in zero-g. One way to form drops under low-g conditions would be to vibrate the substrate supporting the drop liquid. This paper presents a theoretical analysis of the situation in which a vertical rod which supports an initially quiescent pendant drop is impulsively set into oscillations of sufficiently large amplitude along its axis so that a portion of it detaches or is ejected from the rest of the liquid that is left behind on the rod.

Forced oscillations of pendant and sessile drops sans breakup have been studied extensively because they exhibit rich non-linear behavior and also are important in applications. For example, oscillations of pendant drops ${ }^{9}$ and also of liquid bridges ${ }^{10}$ can be used to infer the surface tension and viscosity of the drop liquid.

Formation of drops from fine capillaries through which liquid is forced at a constant flow rate has also been extensively studied experimentally, $7,8,11$ by means of one-dimensional approximations, 7,12 and computationally in the inviscid, irrotational flow limit, ${ }^{13}$ in the Stokes flow limit, ${ }^{14}$ and at arbitrary Reynolds numbers. 15 These studies have shown that as the drop is nearing breakup, a long thread or neck of liquid connects the about to form primary drop from the rest of the liquid hanging from the capillary. The thread first breaks at its downstream end and its tip is accelerated toward the drop that remains hanging from the tube. However, before the thread can recoil entirely and become absorbed by the liquid hanging from the tubc, it breaks at its upstream end and gives rise to one or more satellite droplets. The satellite droplets are undesirable in virtually all applications. In ink-jet printing, for example, they can lead to stray marks on the paper and reduce print quality. Therefore, it is desirable to come up with ways to suppress the formation of satellite droplets. $^{16}$

When a drop that is hanging from or sitting on the tip of a solid rod is forced to undergo time periodic oscillations along its axis and sufficient time is allowed for the drop to reach a steady oscillatory state, the drop deformation is maximized at a number of values of the forcing frequency known as the resonance frequencies for a fixed value of the forcing amplitude. When the forcing amplitude is sufficiently large, the drop should no longer oscillate and instead should undergo breakup. The equations governing the physics of drop oscillations and breakup are summarized in section 2. A powerful finite element algorithm that can go behind earlier works 9,10 and follow drops all the way to breakup is presented in section 3. Results and conclusions are taken up in sections 4 and 5 . 


\section{PROBLEM STATEMENT}

The system, shown in Figure 1, is an axisymmetric drop of an incompressible Newtonian liquid of constant volume $V_{0}$, density $\rho$ and viscosity $\mu$ which is pendant from a solid circular rod of radius $R$ and surrounded by a dynamically inactive ambient gas. The drop/ambient gas interface has constant surface tension $\sigma$. As in Wilkes and Basaran, ${ }^{9}$ the rod is impulsively set into motion along its symmetry axis and sinusoidally in time with forcing amplitude $A R$ and frequency $\Omega \sqrt{\sigma / \rho R^{3}}$. The three-phase drop/rod/ambient gas contact line is constrained to remain at the outer sharp edge of the rod face as the drop deforms. As time advances, the response of the drop surface $S(t)$ will be either (a) attainment of a steady oscillatory state, or limit cycle, once the initial transients decay, or (b) ejection of a primary drop from the rest of the liquid which will remain on the rod surface.

To cast the problem in dimensionless form, the rod radius $R$ is chosen as a characteristic length scalc and the capillary time $t_{c} \equiv \sqrt{\rho R^{3 / \sigma} \sigma}$ is chosen as a time scale. ${ }^{17,18}$ The corresponding velocity and pressure/ stress scales are then $\sqrt{\sigma / \rho R}$ and $\sqrt{\sigma \mu^{2} / \rho R^{3}}$, respectively. The dimensionless groups governing the motion of the liquid are the Reynolds number $R e \equiv \sqrt{\sigma \rho R / \mu}$, the gravitational Bond number $\mathrm{G} \equiv \rho g R^{2} / \sigma$, dimensionless forcing amplitude $A$, dimensionless forcing frequency $\Omega$, and dimensionless volume $V_{d} R^{3}$. Therefore, the instantaneous position of the rod relative to its initial position is given by $z^{\prime}(t) A \sin \Omega t$. The equations are solved in a noninertial frame of reference, in which the origin of the cylindrical coordinate system $(r, z, \theta)$ moves with the rod as it oscillates. The motion of fluid within the drop is governed by the Navier-Stokes system,

$$
\begin{aligned}
& \nabla \cdot \underline{\mathbf{v}}=0 \\
& \operatorname{Re}\left(\frac{\mathrm{D} \underline{\mathbf{v}}}{\mathrm{D} t}+\underline{\mathbf{F}}\right)=\nabla \cdot \underline{\underline{\mathbf{T}}}+\operatorname{ReG} \mathbf{e}_{z}
\end{aligned}
$$

In Equation (2), $\mathbf{E} \equiv-A \Omega^{2} \sin \Omega t \mathbf{e}_{z}$ is the fictitious acceleration term which arises because of the use of a noninertial frame of reference, $\mathbf{e}_{z}$ is a unit vector in the downward $z$-direction, and the dimensionless stress tensor for a Newtonian fluid is given by $\underline{T}=$ $-p \underline{\underline{\mathbf{I}}}+\left[\nabla \underline{\mathbf{v}}+\nabla \underline{\mathbf{y}}^{T}\right]$. The drop shape, which is unknown a priori, is determined by using the kinematic condition as an additional governing equation,

$$
\underline{\mathbf{n}} \cdot\left(\underline{\mathbf{v}}-\underline{\mathbf{y}}_{\mathrm{s}}\right)=0 \text { on } S(t)
$$

where $\mathbf{n}$ is the outward unit normal vector to the surface and $\mathbf{v}$ and $\underline{\mathbf{y}}_{s}$ are velocities of points just inside and on the frec surface $S(t)$, respectively. The other boundary conditions are axisymmetry about the $z$-axis, no slip and no penetration along the rod surface, the balancing of the normal stress by the capillary pressure and the vanishing of the shear stress along the free surface, and the fixed contact line.

Initially, the drop shape is a hemisphere and the fluid is quiescent; rod motion is impulsively commenced at $t=0$.

\section{COMPUTATIONAL METHOD}

The transient system of governing equations (1)-(3) subject to the boundary and initial conditions stated above are solved numerically by a method of lines using the Galerkin/Finite Element Method (G/FEM) for spatial discretization and an adaptive, implicit finite difference method for time discretization. The Galerkin weighted residuals of these equations are solved for the velocity, pressure, and free surface shape by Newton iteration at each time step. Time integration is achieved using a backward-difference predictor for the first four time steps and for four time steps following each remeshing (see below), and a trapezoid-rule integrator and second-order Adams-Bashforth predictor at all other time steps. In the latter case, time step sizes are chosen adaptively such that the time truncation crror does not exceed $10^{-3}$.

The problem domain is divided into elements using one of three methods15. Method 1, Figure 2(a), consists of a central fixed cylindrical region of radius $\sim r$ extending from the rod to a distance $\sim \mathrm{z}$ below it along the centerline, which is surrounded by outer regions which deform with the drop surface. The outer regions' clements are separated by fixed spines and by curves which deform in proportion to the moving surface. Method 2, Figure 2(b), consists of the same regions and a neck region between the rod and the drop regions of Method 1. The spines in this new region are horizontal and spaced at intervals which become smaller as the surface curvature increases. Method 3, Figure 2(c), is identical to Method 2 except that the spine angles in the neck region are varied so as to enable discretization of a portion of the interface which is about to overturn. Method 1 is used for the initial stages of drop oscillation until the minimum neck radius $R_{m}$ reaches a specified value; at that point, Method 2 is used until the slope of the interface near the point of breakup becomes so high that Method 3 is required. Once Method 3 is invoked, no further remeshing occurs. The accuracy of this computational algorithm was confirmed by comparison of results obtained to those reported by Wilkes and Basaran ${ }^{9}$.

Periodically, the mesh structure is reconstructed so as to conform to the present drop shape: this is referred to as remeshing. At various increments of the drop length $L$ or when the minimum neck radius $R_{m}$ approaches the present fixed region boundary $\delta_{r}$ new values of $\delta_{r}$ and $\delta_{z}$ are chosen based on the present drop 
shape. When Method 2 is used, the neck region length $\delta_{y}$ and the number of spines in this region are also recalculated. Once the new elements are located, all unknowns are interpolated from the old mesh onto the new mesh using the relevant basis functions, and time integration is resumed. When $R_{m}$ falls below 0.003 , the primary drop is considered to have detached.

\section{RESULTS AND DISCUSSION}

Figure 3 illustrates the response of a drop for a typical situation in which drop ejection occurs. In this case, $R e=20, G=0, \Omega=3.5$, and $A=0.50$. When the forcing amplitude is lower but the other parameters are the Figure 3 illustrates the response of a drop for a typical situation in which drop ejection occurs. In this case, $R e=20, G=0, \Omega=3.5$, and $A=0.50$. When the forcing amplitude is lower but the other parameters are the same, the primary resonance frequency is known to be about $3.5{ }^{9}$ Figure 3 shows that during the first oscillation period after rod motion begins, the maximum clongation of the drop is considerably lower than that for each subsequent period, in accord with the results of Wilkes and Basaran. ${ }^{9}$ During the second period, however, the drop tip nearly touches the rod and then moves farther away from it than in the first period. As the tip recedes once more, the interface necks rapidly and the drop starts breaking into two distinct fluid masses. In contrast to drop formation from a tube, ${ }^{13,15}$ the primary drop is now moving upward toward the neck while the rod is accelerating the liquid in its vicinity toward the neck. As shown in Figure 4 at a time step near ejection, the down moving fluid near the rod and the up moving fluid in the bulk of the primary drop work in unison to prevent drop breakup. However, by this time the capillary pressure in the thin neck is so high that evacuation of the neck prevails over the former mechanism. Moreover, on account of the receding primary drop and the down moving rod, the interface above the neck overturns and becomes a multi-valued function of $z$. The manner in which the interface breaks is thus quite different from that observed for drop formation from a tube: Because a long thread does not form, the likelihood of satellite drop formation is minimized.

A similar series of computations was performed using the same parameters as in Figure 3 except for $A$, which was varied from 0.2 to 0.3 . These computations showed that the critical forcing amplitude $A_{c}$ for ejection under these conditions was $0.274 \pm 0.001$. For cases in which $A<A_{c}$, the drops oscillated without ejection, whereas when $A>A_{c}$, ejection was observed and the final drop shapes (not shown) were remarkably similar - the most notable variation in drop shape over this range of amplitudes was the extent of interface overturning, which increases with $A$. In each of these cases, the time of drop ejection, when it occurred, was near the end of the second rod oscillation period, except for the case with $A=0.274$ when it occurred during the fifth period. Because the drop is at rest at the start of the oscillations, a considerably higher forcing amplitude is necessary to effect ejection in a single period. By contrast, ejection after two or more periods appears to occur only when $\mathrm{A}$ is equal to or slightly higher than $A_{c}$. 


\section{CONCLUSIONS AND OUTLOOK}

The computational method used in this paper can accurately account for the fluid dynamics of drop ejection from an oscillating rod. According to the foregoing results, the interface develops an infinite slope before the radius of the thread or the neck vanishes. Therefore, recently popularized one-dimensional slender-jet equations that represent interface shape as a function of axial distance alone ${ }^{7,12,14}$ cannot be used to model the dynamics of drop ejection.

Suppression of neck formation, in contrast to situations when a liquid is made to flow at a constant flow rate through a capillary, is an unexpected but highly desirable outcome of the technique of drop production considered here. Exploitation of this finding in practical applications such as ink-jet printing is pending.

\section{ACKNOWLEDGMENTS}

This research was sponsored by the NASA Microgravity Fluid Physics Program. EDW was also supported in part by a Shell Doctoral Dissertation Fellowship. 


\section{REFERENCES}

[1] P.J. Bailes and A. Winward, "Progress in liquid-liquid cxtraction," Trans. Instr. Chem. Engrs. 50, 240 (1972).

[2] W.J. Heideger and M.W. Wright, "Liquid extraction during drop formation: effect of formation time," AIChE J. 32, 1372 (1986).

[3] T.W. Shicld, D.B. Bogy, and F.E. Talke, "Drop formation by DOD ink-jet nozzles: A comparison of experiment and numerical simulation," IBM J. Res. Dev. 31, 96 (1987).

[4] I. Rezanka and R. Eschbach, "Recent Progress in Ink Jet Technologies," (IST Press, Springfield, VA, 1996).

[5] C.A. Macleod and C.J. Radke, "A growing drop technique for measuring dynamic interfacial tension," J. Colloid Interface Sci. 160, 435 (1993).

[6] X. Zhang, M.T. Harris, and O.A. Basaran, "Measurement of dynamic surface tension by a growing drop technique," J. Colloid Interface Sci. 168, 47 (1994).

[7] X.D. Shi, M.P. Brenner, and S.R. Nagel, "A cascade of structure in a drop falling from a faucet," Science 265 , 219 (1994).

[8] X. Zhang and O.A. Basaran, "An experimental study of dynamics of drop formation," Phys. Fluids 7, 1184 (1995)

[9] E.D. Wilkes and O.A. Basaran, "Forced oscillations of pendant (sessile) drops," Phys. Fluids 9 (6), 1512 (1997).

[10]T-Y. Chen and J.A. Tsamopoulos, "Non-linear dynamics of capillary bridges: theory," J. Fluid Mech. 255, 373 (19).

[11]D.H. Peregrine, G. Shoker, and A. Symon, "The bifurcation of liquid bridges," J. Fluid Mech. 212, 25 (1990).

[12]J. Eggers and T.F. Dupont, "Drop formation in a onedimensional approximation of the Navier-Stokes equation," J. Fluid Mech. 262, 205 (1994).

[13]R.M.S.M. Schulkes, "The evolution and bifurcation of a pendant drop," J. Fluid Mech. 278, 83 (1994).

[14]M.P. Brenner, J. Eggers, K. Joseph, S.R. Nagel, and X.D. Shi, "Brcakdown of scaling in droplet fission at high Reynolds numbers," Phys. Fluids 9, 1573 (1997).

[15]E.D. Wilkes, S.D. Phillips, and O.A. Basaran, "Computational and experimental analysis of dynamics of drop formation," Phys. Fluids (1998, submitted).

[16]X. Zhang and O.A. Basaran, "Dynamics of drop formation from a capillary in the presence of an electric field," J. Fluid Mech. 326, 239 (1996).

[17]O.A. Basaran, "Nonlinear oscillations of viscous liquid drops," J. Fluid Mech. 241, 169 (1992).

[18]O.A. Basaran and D.W. DePaoli, "Non-linear oscillations of pendant drops," Phys. Fluids 6 (9), 2923 (1994). 
E. D. Wilkes and O. A. Basaran
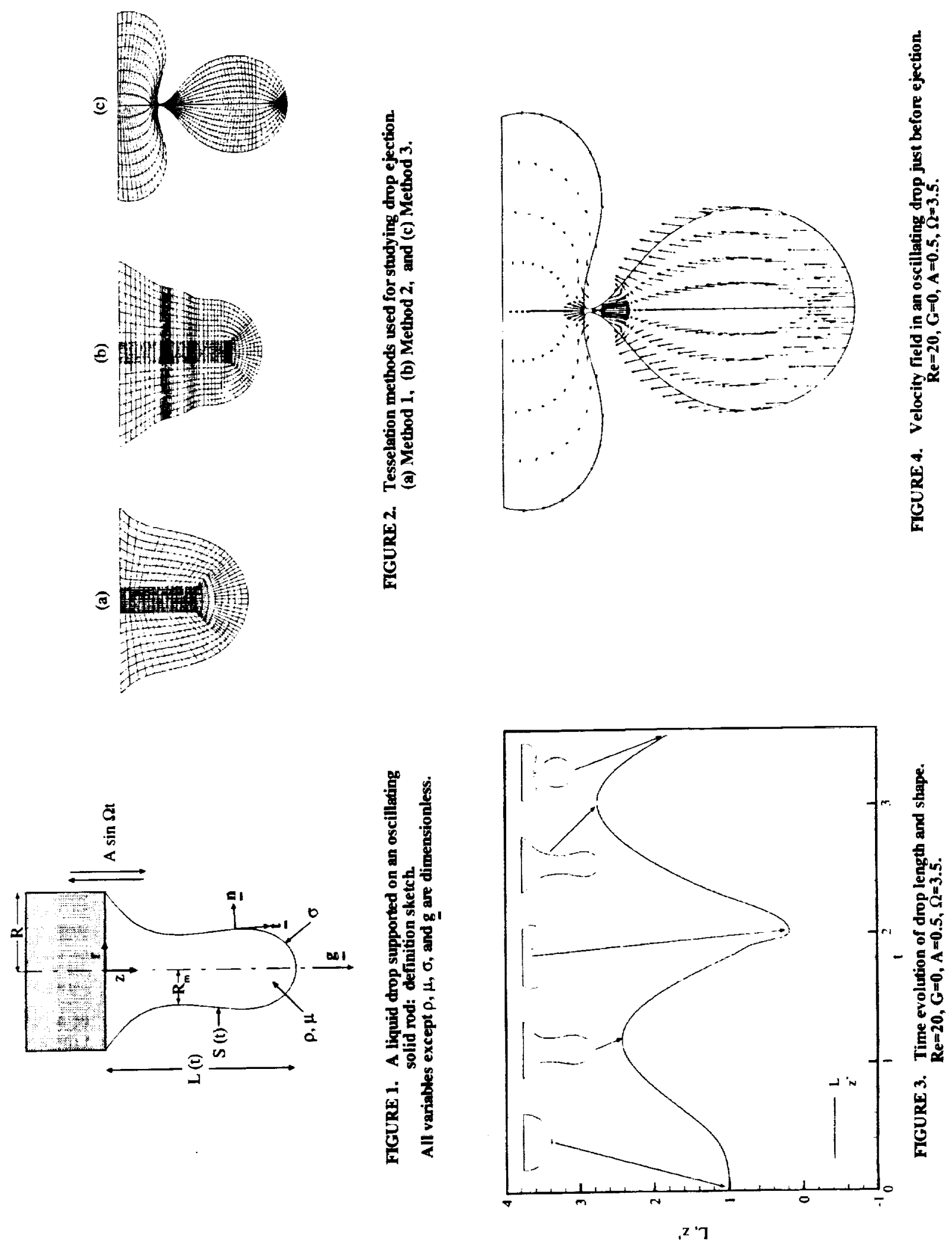